\title{
Clinicopathological Study of Marrow Infiltration by Lymphoproliferative Disorders: A 2-year Experience from a Cancer Institute
} \author{
Fatima Farzeen Fameena ${ }^{3}$ \\ ${ }^{1}$ Department of Pathology, MNJ Institute of Oncology and Regional \\ Cancer Centre, Hyderabad, Telangana, India \\ ${ }^{2}$ Department of Medical Oncology, MNJ Institute of Oncology and \\ Regional Cancer Centre, Hyderabad, Telangana, India \\ ${ }^{3} \mathrm{MNJ}$ Institute of Oncology and Regional Cancer Centre, Hyderabad, \\ Telangana, India \\ Asian J Oncol 2018;4:43-47
}

Sai Mallikarjun ${ }^{1}$ Abid Hussain ${ }^{1}$ Triveni Bhopal ${ }^{1}$ Sree Lakshmi ${ }^{1} \quad$ Sudha Sinha ${ }^{1}$

\begin{abstract}
Address for correspondence Abid Hussain, MBBS, MD, Department of Pathology, MNJ Institute of Oncology and Regional Cancer Centre, Beside Niloufer Hospital, Niloufer Road, Red Hills, Bazar Ghat, Hyderabad 500 004, Telangana, India (e-mail: hussain.abid111@gmail.com).
\end{abstract}

\begin{abstract}
Bone marrow (BM) examination is an essential tool in evaluation and staging of non-Hodgkin lymphoma (NHL). The aim of this study is to overview the approach to the use of unilateral versus bilateral BM sampling in lymphoproliferative disorders for diagnosis and staging. All cases diagnosed as lymphoproliferative disorders from January 2014 to January 2017 were studied. Bilateral trephine biopsy and aspiration of 414 cases were assessed for involvement patterns and presence/absence of background changes. Bone marrow involvement (BMI) was seen in 109 (26.3\%) cases. The cases ranged from 2 to 80 years. BMI was more frequent in B-cell lymphomas than T-cell lymphomas as was the incidence (B-NHL= 87/109 [79.8\%]; T-NHL=21/109 [19.1\%]). Among B-cell lymphomas, BMI was most frequent in chronic lymphocytic lymphoma followed by B-lymphoblastic lymphoma (BLL) and mantle cell lymphoma. Among T-cell lymphomas, BMI was most frequent in T-lymphoblastic lymphoma (TLL). A single case

Keywords

- bone marrow involvement

- chronic lymphocytic lymphoma

- immunohistochemistry

- lymphoma of anaplastic large cell lymphoma (ALCL) had BMI. Bone marrow aspiration (BMA) and bone marrow trephine (BMT) agreed in 97\% of cases ( 401 of 414: 308 both negative, 93 both positive). Additional 13 cases were diagnosed on BMT whereas BMA was negative for these cases. Background changes (fibrosis and necrosis) were common in TLL followed by BLL. Of the 109 positive cases, 4 were missed by unilateral BMT. Peripheral spill of lymphoma was seen in 14 of 109 cases. BMT involvement by lymphoma is more commonly seen in lymphoblastic lymphoma. Bilateral trephine biopsy remains gold standard to determine the marrow involvement and staging and assess the remission.
\end{abstract}

\section{Introduction}

Bone marrow (BM) examination is an essential tool in evaluation and staging of non-Hodgkin lymphoma (NHL) at the time of initial diagnosis as well as after therapy. The documentation of BM involvement results in clinical stage IV, upgrades the international prognostic index (IPI), and affects survival and therapeutic strategies for NHL.
Bilateral iliac crest BM biopsies of 1.5 to $2 \mathrm{~cm}$ length and step sections for morphologic view are mandatory for detection of small focal lesions accompanied by secondary changes such as fibrosis and necrosis that may not be readily detected in aspirate smear preparations, thereby increasing the yield of possible diagnosis. ${ }^{1}$ Several studies regarding the bone marrow involvement (BMI) in NHL are mentioned in the literature. ${ }^{2,3}$ We herein describe in detail
DOI https://doi.org/

10.1055/s-0038-1676765

ISSN 2454-6798.
License terms

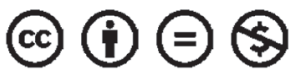


the incidence, morphologic and histopathologic patterns with immunohistochemistry of BMI of NHL along with approach to the use of unilateral versus bilateral BM sampling in lymphoproliferative disorders for diagnosis and staging, which were classified according to the 2016 World Health Organization (WHO) classification of lymphoid neoplasm. To our knowledge, this is the largest case series of BMI by NHL from a developing country.

\section{Materials and Methods}

All cases (414) diagnosed as lymphoproliferative disorders on histomorphology and immunohistochemistry (IHC) from January 2014 to January 2017 were studied. These cases were assessed for the occurrence of BMI and architectural features including involvement pattern (paratrabecular, interstitial [nodular and intrasinusoidal], diffuse and mixed patterns) and presence/absence of background changes (granuloma, stromal fibrosis, necrosis, and eosinophils). Bilateral BM biopsies were obtained using the conventional technique with a Jamshidi needle from the posterior superior iliac spines under local anesthesia in all the cases for staging workup. The biopsies were fixed in $10 \%$ formalin solution and decalcified using 10\% Formic acid and 5\% Formaldehyde solution for 4 to 6 hours, followed by routine processing and paraffin embedding. ${ }^{4}$ Serial sections of 3 to $4 \mu \mathrm{m}$ thickness were cut and stained by hematoxylin and eosin (H\&E) and supplemented with immune stains. Sections with at least five well-preserved marrow spaces were studied for cellularity, normal hematologic elements, presence of infiltration, histologic pattern and morphology of infiltration, fibrosis, and other secondary changes. The clinical details were obtained from the medical records. IHC panel for low-grade lymphomas with small- to medium-sized cells (chronic lymphocytic lymphoma [CLL], follicular lymphoma [FL], mantle cell lymphoma [MCL], marginal zone lymphoma [MZL]) included CD5, CD10, CD23, bcl2, cyclin D1, CD20, and Ki67. For high-grade lymphomas with large cells (diffuse large B-cell lymphoma [DLBCL], FL), the panel included CD20, CD3, CD5, CD10, bcl2, CD138, CD34, TdT, and Ki67. Similarly, for blastic lymphomas (B-lymphoblastic lymphoma [BLL], T-lymphoblastic lymphoma [TLL], MCL), the panel comprised CD20, CD3, CD10, CD 34, TdT, cyclin D1, and Ki67. Cheson's criteria $^{5}$ were followed, and BM was reported as

- Positive or negative for involvement

- Pattern of infiltration: Paratrabecular, interstitial (nodular and intrasinosoidal), diffuse and mixed patterns

- Extent of infiltration: Semiquantified $-<50 \%$ and $>50 \%$

- Presence of small or large cells.

\section{Results}

\section{Demographic and Clinical Aspects}

During the study period, 414 cases of lymphoproliferative disorders were diagnosed at our institute. These cases included 325 NHL cases and 89 cases of Hodgkin's lymphoma (HL). All these cases had BM biopsy for staging workup. Of these, 109 cases had BMI (B-NHL = 87 [79.8\%]; T-NHL = 21 [19.1\%]; HL = 1 [0.1\%]). The cases included age range from 2 to 80 years, with a median age of 42 years. There was a male predominance with male-to-female ratio of 1.5:1. Lymphadenopathy was the most common presentation, followed by extranodal involvement in 49 cases.

\section{Histopathologic Aspects}

Histopathology slides were reviewed in all the cases. Among B-cell lymphomas, BMI was most frequent in CLL (29/87) $34.4 \%$, followed by LL (16/87) $18.3 \%$, and MCL (11/87) $13 \%$. A single case of BL had BMI (1/87) 1.1\%. Among all the B-cell lymphomas, the lowest incidence of BMI was seen in high-grade lymphomas such as DLBCL 5.6\% (7/125) and Burkitt's lymphoma 5\% $1 / 20)$. The incidence of BMI in all the subtypes of $\mathrm{NHL}$ in relation to $\mathrm{WHO}$ classification is shown in - Table 1. Among T-cell lymphomas, BMI was most frequent in lymphoblastic lymphoma (LL). A single case of anaplastic large cell lymphoma (ALCL) had BMI. Peripheral blood involvement was seen in 14/109 cases.

Bone marrow aspiration (BMA) and bone marrow trephine (BMT) agreed in 97\% of cases (401 of 414: 308 both negative, 93 both positive). In the remaining $3 \%, 13$ cases were positive on BMT and negative on BMA.

The predominant histologic pattern of involvement by a lymphomatous infiltrate was mixed (48.6\%, 53/109), followed by diffuse (44\%, 48/109) and interstitial $(7.3 \%, 8 / 109)$ (-Figs. 1, 2).

Of the mixed pattern, 28 of the 53 cases had predominantly interstitial involvement and comprised mainly DLBCL (6 cases), FL (2 cases) and CLL (20 cases). Four of the five FL cases showed predilection for paratrabecular area

Table 1 Comparison of incidence of lymphoma in this study with other studies

\begin{tabular}{|l|l|l|}
\hline $\begin{array}{l}\text { Type of } \\
\text { lymphoma }\end{array}$ & $\begin{array}{l}\text { Percentage } \\
\text { occurrence } \\
\text { (involved/all) in this } \\
\text { study }\end{array}$ & $\begin{array}{l}\text { Percentage } \\
\text { occurrence } \\
\text { (involved/all) }\end{array}$ \\
\hline BLL & $100(19 / 19)$ & $42.9(3 / 7)$ \\
\hline TLL & $100(19 / 19)$ & $34.9(15 / 43)$ \\
\hline CLL & $100(30 / 30)$ & $78.6(22 / 28)$ \\
\hline FL & $30(5 / 17)$ & $41(10 / 24)$ \\
\hline MCL & $60(12 / 20)$ & $48.1(28 / 57)$ \\
\hline MZL & $38(6 / 16)$ & $58(10 / 17)$ \\
\hline DLBCL & $5.6(7 / 125)$ & $6.4(22 / 346)$ \\
\hline BL & $5(1 / 20)$ & $0(0 / 7)$ \\
\hline HDL & $1.1(1 / 89)$ & $4.4(6 / 138)$ \\
\hline $\begin{array}{l}\text { T-cell } \\
\text { lymphoma/ } \\
\text { ALCL }\end{array}$ & $12.5(1 / 8)$ & $6.3(1 / 16)$ \\
\hline Abbev: & & \\
\hline
\end{tabular}

Abbreviations: ALCL, anaplastic large cell lymphoma; BL, Burkitt's lymphoma; BLL, B-lymphoblastic lymphoma; CLL, chronic lymphoblastic lymphoma; DLBCL, diffuse large B-cell lymphoma; FL, follicular lymphoma; HDL, Hodgkin's disease lymphoma; MCL, mantle cell lymphoma; MZL, marginal zone lymphoma; TLL, T-lymphoblastic lymphoma. 


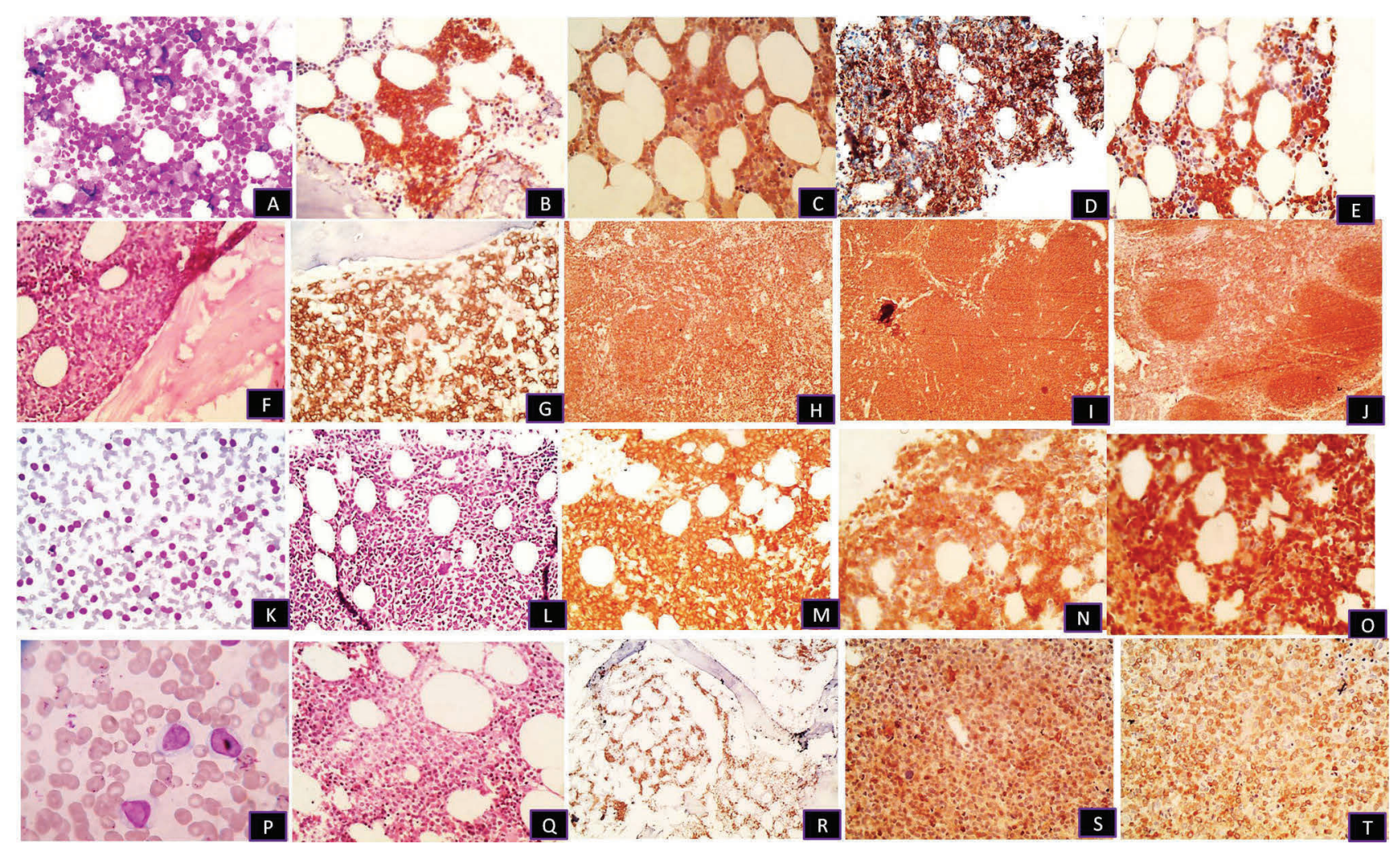

Fig. 1 (A, B) Trephine biopsy, interstitial pattern in chronic lymphoblastic lymphoma on H\&E and CD20 (×100). (C-E) Same case positive for CD5, CD23, and bcl2 $(\times 100)$. (F, G) Paratrabecular pattern in follicular lymphoma with H\&E and CD20 ( $\times 100)$. (H-J) Immunohistochemistry with $\mathrm{CD} 10$ in bone marrow and CD20, bcl2 in lymph node, respectively $(\times 100)$. (K-0) Peripheral blood spill and interstitial pattern in mantle cell lymphoma on H\&E, CD20, CD5, and cyclin D1 (×100). (P-T) Peripheral spill with interstitial and sinusoidal pattern in marginal zone lymphoma on H\&E, CD20, CD10, and bcl2 (×400) $(\times 100)$.

with paratrabecular only pattern in two cases. The other lymphomas showing paratrabecular pattern were MCL, MZL, and peripheral T-cell (PTC) lymphoma. Interstitial pattern was more frequent in CLL and MCL. Diffuse, interstitial, and focal patterns were seen in T-cell neoplasms. Focal nodular pattern was found in most lymphomas, either as a distinct pattern or a part of mixed pattern.

A discordant histology between BM and primary anatomic site (lymph node) was found in 28.5\% (2/7) of the DLBCL cases. Discordance was not seen in any other type of lymphoma.

\section{Bone Marrow Background Changes}

The background changes of 109 involved BMT included necrosis ( 8 cases), eosinophilia ( 5 cases), granuloma ( 2 cases), and fibrosis (20 cases) (-Table 2 ).

\section{Discussion}

BM examination is an important procedure in the diagnosis and management of patients with lymphoma. BMI by malignant lymphoma indicates stage IV disease, and hence bilateral BM biopsy was recommended with trephine biopsy being preferred to BMA for detecting marrow infiltration. ${ }^{6}$ The incidence and progression to BMI varies according to various histologic subtypes of lymphoma. We used the 2016 WHO classification of lymphoid neoplasm to classify the histology on the primary anatomic site for correlating with the marrow histology.
In our study, BMI was found in all age groups with no obvious sex differences. Different patterns of BM infiltration are described in lymphomas: paratrabecular, interstitial, diffuse, and mixed. Mixed pattern was more common in our study, which was similarly seen by Arber and George. ${ }^{7}$ However, this varied from the findings of Foucar et al, who showed predominantly focal pattern (70\%). ${ }^{8}$ The difference could be due to the larger number of cases studied by them and the fact that there were more FLs in their study. Marrow infiltration suggests a poor prognosis, especially the diffuse pattern $^{9}$ that was seen in $44.4 \%$ (48/108) of the cases.

In this study, BM was involved by lymphoma in $26.3 \%$ (109/414) cases. Other studies have described a considerable variation in the BMI in lymphoproliferative disorders, ranging between 29.6 and $53 \% .^{10}$ The variation in previous studies and the present one can be due to inclusion of unequal proportions of patients with early and advanced disease. In addition, the inclusion of different proportions of various histologic subtypes of lymphomas in the different studies may account for this variation as the incidence of BM pathology varies greatly according to the histological subtypes.

Incidence of BMI was high in $\operatorname{LL}(100 \%$ in $B L L=16 / 16 ; 100 \%$ in TLL $=19 / 19)$ and CLL $(100 \%$ [30/30]), while the incidence of BMI was very low in high-grade lymphomas such as DLBCL 5.6\% (7/125) and Burkitt's lymphoma 5\% (1/20), which was similarly seen in a study by Shi et al. ${ }^{11}$ Incidence of BMI in MCL was $60 \%$ (12/20), which was relatively higher 

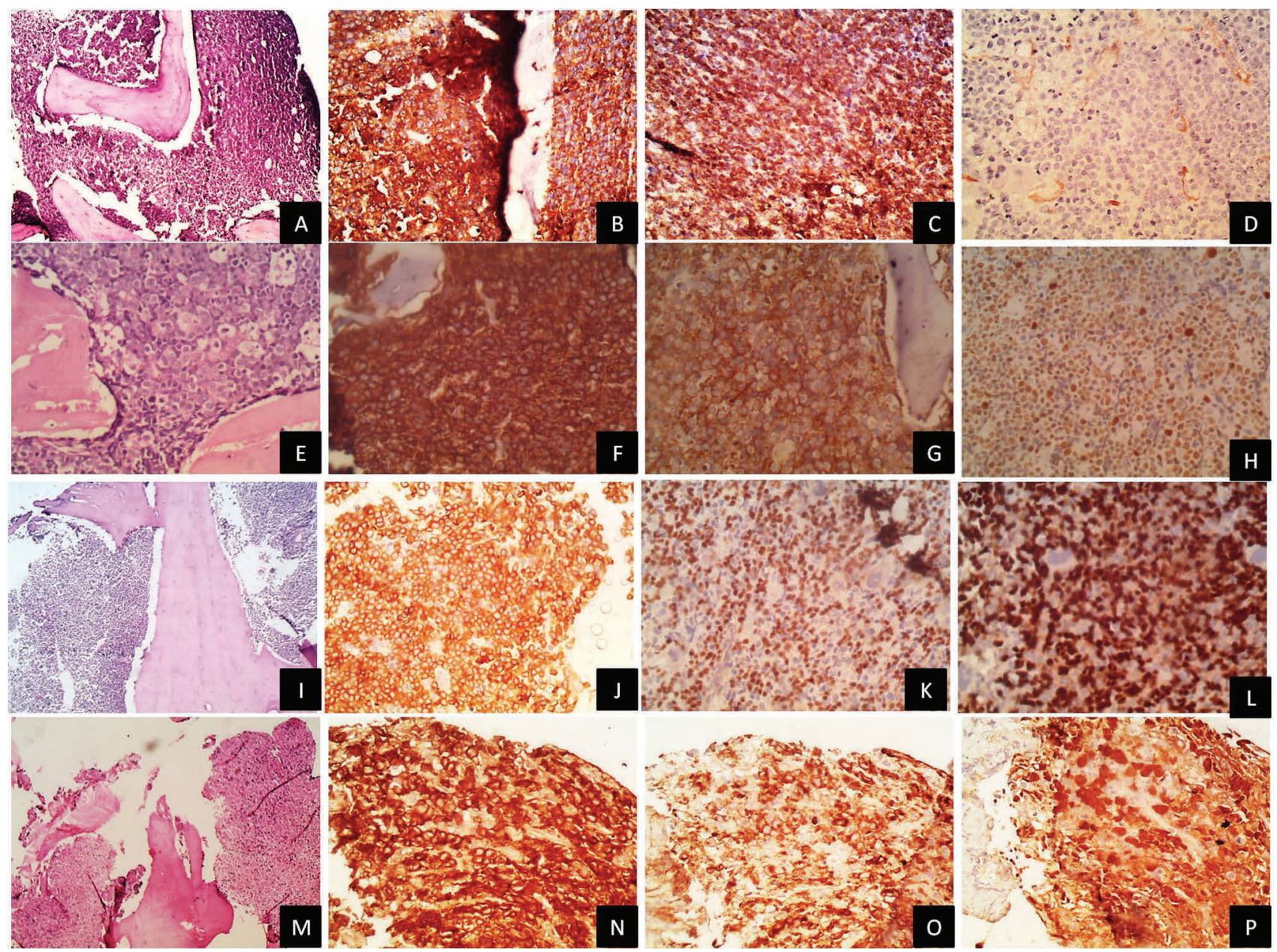

Fig. 2 (A, B) Trephine biopsy diffuse pattern in diffuse large B-cell lymphoma on H\&E and CD20 (×100). (C, D) bcl2 and Tdt, respectively (×100). (E, F) Diffuse pattern with starry sky appearance in Burkitt's lymphoma on H\&E and CD20 (×200). (G, H) CD10 and high $\mathrm{K}_{\mathrm{i}} 67$ labeling index (×100). (I, J) Diffuse pattern in BLL on H\&E and CD20 (×100). (K, L) PAX5 and Tdt positivity, respectively $(\times 100)$. (M, N) Diffuse pattern in anaplastic large cell lymphoma on H\&E and CD30 immunohistochemistry, respectively $(\times 100)$. (0, P) Confirmed with positivity for Epithelial Membrane Antigen (EMA) and anaplastic lymphoma kinase immunohistochemistry, respectively $(\times 100)$.

Table 2 Secondary changes seen in bone marrow biopsies

\begin{tabular}{|l|l|l|l|l|}
\hline Type of lymphoma & Necrosis $(\boldsymbol{n}=5)$ & Fibrosis $(\boldsymbol{n}=\mathbf{2 0})$ & Eosinophilia $(\boldsymbol{n}=\mathbf{5})$ & Granuloma $(\boldsymbol{n}=\mathbf{2})$ \\
\hline BLL & 1 & 7 & - & \\
\hline TLL & 2 & 9 & - & \\
\hline CLL & - & - & 3 & \\
\hline FL & - & - & - & \\
\hline MCL & - & 1 & - & \\
\hline MZL & - & - & - & \\
\hline DLBCL & 1 & 3 & - & 1 \\
\hline BL & - & - & - & 1 \\
\hline HDL & - & - & 1 & \\
\hline T-cell lymphoma/ALCL & 1 & - & 1 & \\
\hline
\end{tabular}

Abbreviations: ALCL, anaplastic large cell lymphoma; BL, Burkitt’s lymphoma; BLL, B-lymphoblastic lymphoma; CLL, chronic lymphoblastic lymphoma; DLBCL, diffuse large B-cell lymphoma; FL, follicular lymphoma; HDL, Hodgkin's disease lymphoma; MCL, mantle cell lymphoma; MZL, marginal zone lymphoma; TLL, T-lymphoblastic lymphoma. 
83\% (39/46) in Cohen et al. ${ }^{12}$ The reason is less number of cases and patients diagnosed at an early phase of disease in our study. Incidence of BMI of ALCL was 12.5\% (1/8), which was similarly shown as $19 \%$ (8/42) by Fraga et al. ${ }^{13}$ Incidence of BMI in $\mathrm{HL}$ in our study was $1.1 \%$ (1/89).

Concordant BMI is defined as similar morphology of cells in both primary site and bone marrow. Large cells in DLBCL is a concordant finding and is associated with poorer prognosis and increased risk of central nervous system (CNS) relapse. Discordant BMI is defined as discordance in the morphology of cells in bone marrow when compared with the primary site. A discordant histology between the BM and the primary anatomic site was encountered in $28.5 \%$ (2/7) of the DLBCL cases, which is similar to 16 to $40 \%$ reported in the literature with this same histologic subtype of lymphomas. Discordance was not seen in any other type of lymphomas in our study.

\section{Conclusion}

This study highlights the incidence and different patterns of BMI, background changes, and discordance between BM morphology and primary site histology. A discordant histology between BM and primary anatomic site was found in $28.5 \%(2 / 7)$ of the DLBCL cases.

BMA and BMT agreed in $97 \%$ of cases ( 401 of 414: 308 both negative, 93 both positive). BMT biopsy showed involvement of lymphoma in $26.3 \%$ cases, with unilateral positivity in only four cases. Therefore, bilateral and generous BM biopsy should be performed routinely for adequacy and to identify tiny focal involvement, thereby increasing the yield of possible diagnosis.

\section{Funding}

None.

\section{Conflict of interest}

None declared.

\section{Acknowledgment}

The authors would like to acknowledge the support from technicians of department of histopathology.

\section{References}

1 Brunning RD, Bloomfield CD, McKenna RW, Peterson LA. Bilateral trephine bone marrow biopsies in lymphoma and other neoplastic diseases. Ann Intern Med 1975;82(3):365-366

2 Conlan MG, Bast M, Armitage JO, Weisenburger DD; Nebraska Lymphoma Study Group. Bone marrow involvement by non-Hodgkin's lymphoma: the clinical significance of morphologic discordance between the lymph node and bone marrow. J Clin Oncol 1990;8(7):1163-1172

3 Jeong SY, Chang YH, Lee JK, Hong YJ, Hong SI, Lee SS. [Incidence and histologic patterns of bone marrow involvement of malignant lymphoma based on the World Health Organization classification: a single institution study] [in Korean]. Korean J Lab Med 2007;27(6):383-387

4 Culling CF, Allison RT, Barr WT. Connective tissue. In: Cellular Pathology Techniques. 4th ed. London, UK: Butterworth and Co.; 1985:172-173

5 Chen T, McDonald A, Shadbolt B, Talaulikar D. Precision of histological bone marrow staging in follicular lymphoma and diffuse large B-cell lymphoma. Clin Invest Med 2012;35(6):E358-E364

6 Bain BJ, Clark DM, Lampert IA, Wilkins BS, eds. Bone Marrow Pathology. 3rd ed. Oxford: Blackwell Science; 2001

7 Arber DA, George TI. Bone marrow biopsy involvement by non-Hodgkin's lymphoma: frequency of lymphoma types, patterns, blood involvement, and discordance with other sites in 450 specimens. Am J Surg Pathol 2005;29(12):1549-1557

8 Foucar K, McKenna RW, Frizzera G, Brunning RD. Bone marrow and blood involvement by lymphoma in relationship to the Lukes-Collins classification. Cancer 1982;49(5):888-897

9 Schmid C, Isaacson PG. Bone marrow trephine biopsy in lymphoproliferative disease. J Clin Pathol 1992;45(9):745-750

10 Juneja SK, Wolf MM, Cooper IA. Value of bilateral bone marrow biopsy specimens in non-Hodgkin's lymphoma. J Clin Pathol 1990;43(8):630-632

11 Shi YF, Li XH, Song YQ, Song W, Lai YM. Involvement of bone marrow in lymphoma: pathological investigation in a single-center from northern China. Int J Clin Exp Pathol 2015;8(6):7102-7111

12 Cohen PL, Kurtin PJ, Donovan KA, Hanson CA. Bone marrow and peripheral blood involvement in mantle cell lymphoma. Br J Haematol 1998;101(2):302-310

13 Fraga M, Brousset P, Schlaifer D, et al. Bone marrow involvement in anaplastic large cell lymphoma. Immunohistochemical detection of minimal disease and its prognostic significance. Am J Clin Pathol 1995;103(1):82-89 